\title{
Olanzapine-Induced Tardive Blepharospasm: A Case Report
}

\author{
Yih Chew Poh ${ }^{1}$, Suzaily Wahab ${ }^{1,{ }^{*}}$, Yoong Mei Theng ${ }^{1}$, Arunakiri Muthukrishnan ${ }^{2}$ and Kalaivani \\ Murugan $^{2}$ \\ ${ }^{1}$ Psychiatry Department, Universiti Kebangsaan Malaysia Medical Centre (UKMMC), Cheras, Malaysia \\ ${ }^{2}$ Psychiatry Department, Hospital Bahagia Ulu Kinta, Tanjung Rambutan, Malaysia \\ "Corresponding author: Psychiatry Department, Universiti Kebangsaan Malaysia Medical Centre, Cheras, , Malaysia. Tel/Fax: +60-391455555, Email: suzailywhb@yahoo.com
}

Received 2019 February 27; Revised 2019 July 30; Accepted 2019 August 08.

\begin{abstract}
Introduction: The objective of this case report was to highlight one of the uncommon subtypes of tardive dyskinesia (TD) as tardive blepharospasm secondary to olanzapine.

Case Presentation: We reported a rare case of young-onset schizophrenia in an adolescent patient, who was treated with olanzapine, but subsequently developed tardive blepharospasm. Clinical resolution of the blepharospasm after olanzapine was stopped and switched to quetiapine.

Conclusions: Tardive blepharospasm can be a regarded as a presentation of tardive dyskinesia. Complete recovery of the symptoms can be achieved by stopping the offending drug early and switching to a serotonin-dopamine receptor antagonist.
\end{abstract}

Keywords: Antipsychotic, Blepharospasm, Drug-Induced Dyskinesia, Olanzapine, Tardive Dyskinesia

\section{Introduction}

Tardive dyskinesia (TD) is a delayed-onset movement disorder, which is characterized by repetitive, spasmodic and patterned muscle contraction. Exposure to longterm antipsychotics is one of the known causes for TD (1). It is estimated that about one-third of outpatients with schizophrenia treated with antipsychotics has experienced TD (2). Younger age is associated with a higher risk of TD (3). There are few recognized subtypes of TD, including choreoathetosis, tardive dystonia, blepharospasm, and tardive akathisia. Blepharospasm is characterized by the repetitive, forceful, and sustained involuntary contractions of the orbicularis oculi $(4,5)$. Blepharospasm can be uncommonly presented as the only sign of TD (5). The symptoms can be unpleasant and distressing to the patients. We reported a case of olanzapine-induced tardive blepharospasm in an adolescent patient diagnosed with the young-onset schizophrenia.

\section{Case Presentation}

This case report depicts a 17-year old Malay male patient diagnosed with young-onset schizophrenia, who had been treated in a mental health institution due to poor family support. He was treated with oral olanzapine (20 mg) taken daily for the past two years without any side effects, and he was in partial remission with residual psychotic symptoms. During one of the reviews, he reported blepharospasm, characterized by excessive forceful blinking on both of his eyelids. Further interviews revealed that he had difficulty to keep his eyes open due to the excessive forceful blinking that he had experienced. He reported feeling of discomfort over both his eyes and the blepharospasm had affected his daily activities, as he has spent a longer amount of time in the bed during the daytime resting and has closed his eyes. The severity of blepharospasm alleviated when he was asleep and exacerbated when in stressful situations. He was neither used anticholinergic medication nor any other medications that are known to cause dystonia or blepharospasm.

He had normal birth and early developmental milestones. His academic performance was below the average before he dropped out of the school at the age of 14 years. He was diagnosed with early-onset schizophrenia at the age of 15 years. He had neither a history of blepharospasm nor movement disorders and also had no family history of such disorders. The patient was treated with risperidone before it was switched to olanzapine, due to poor response to risperidone. He was previously treated with risperidone for four months, and risperidone dosage was titrated up to $4 \mathrm{mg}$ daily.

On mental state examination, he was concerned with the blepharospasm and had difficulty to maintain eye con- 
tact during the interview. He experienced residual third person auditory hallucinations, however there were no active perceptual disturbances during examination. Physical examination revealed that he had spasm over his bilateral eyelids, which was characterized by frequent and excessive blinking, at a rate of about 50 to 60 times per minute. Neurological examination did not reveal any abnormalities other than blepharospasm. No other involuntary movements were noted during physical examination. An initial eye assessment and fundus examination were done by a trained medical assistant at the ophthalmology clinic of the hospital and the results showed no significant abnormality except for the bilateral blepharospasm. Routine blood investigation results were normal. He was then given an early appointment to visit the ophthalmologist clinic. The hospital's physician was consulted and other secondary causes of blepharospasm were improbable. A provisional diagnosis of olanzapine-induced tardive blepharospasm was made clinically based on the clinical history and physical examination.

Dosage of olanzapine was gradually tapered down to $10 \mathrm{mg}$ daily and subsequently to $5 \mathrm{mg}$ daily over two weeks. At the same time, he started taking clonazepam (1 mg) orally per day as recommended by the American Academy of Neurology (AAN) guideline in 2013 for treatment of tardive syndrome (6). The psychiatrist decided to initiate a new antipsychotic and olanzapine was cross-tapered. He started receiving quetiapine (200 $\mathrm{mg}$ ) orally per day concurrently with clonazepam and olanzapine was tapered off gradually. As suggested in the 13th Edition of Maudsley Prescribing Guidelines in Psychiatry, quetiapine may be useful in the alleviation of the tardive syndrome (7). Unfortunately, clonazepam had to be taken off after one week, because he experienced daytime somnolence, likely due to the cross tapering between olanzapine and quetiapine. He had achieved a complete resolution of blepharospasm after olanzapine was taken off for two weeks, whereas quetiapine (200 mg) was continued. There was no worsening of his psychotic symptoms during the cross tapering period. He felt relieved with the resolution of blepharospasm. Psychoeducation regarding the possible side effects was given to him, so that he could inform the therapist swiftly about the side effects in the future. He was monitored closely for any possible recurrence of blepharospasm or other side effects, since he was at the risk of developing similar adverse drug reactions. To date, there is no recurrence of blepharospasm or any form of TD due to quetiapine use.

\section{Discussion}

The search for the most efficacious antipsychotic with the improved drug delivery systems (8) considering the risks for side effects $(9,10)$ has been ongoing, aiming at providing an optimum treatment for psychosis in patients with schizophrenia.

Despite the introduction of atypical antipsychotics, which are known to have a lower incidence of extrapyramidal side effects (EPS) and TD, there are still reported cases of TD (11). Atypical antipsychotics have thought to have a lower incidence of EPS (12), due to its serotonergic blockade, which leads to the release of dopamine to balance out the post-synaptic blockade (13). Among the atypical antipsychotics, olanzapine has a higher risk of developing TD, due to its relatively higher D2 receptor occupancy, which is dose-dependent (14).

Generally, TD is commonly present in elderly patients with long-term antipsychotic use (13). Nevertheless, there is still limited information about TD in adolescent patients. The prevalence of TD associated with antipsychotic usage in children and adolescents generally ranged from one to $4.8 \%$, whereas its prevalence in adults is as high as $20 \%$ (15). A recent study conducted on the pediatric population suggested that the younger age is one of the risk factors for developing TD (3).

Blepharospasm is an ocular symptom presented as involuntary closure of both eyelids $(4,5)$. It is a clinical symptom and must be distinguished regarding the primary and secondary causes. Primary blepharospasm is also known as benign essential blepharospasm (BEB) develops spontaneously and can run in families (16). Secondary blepharospasm is less common than the primary blepharospasm and is usually due to the consumed medications (antipsychotics, dopaminomimetics, dopamine antagonists and antihistamines) (5) or is less commonly occurred secondary to other conditions (such as Parkinson's disease, Wilson's disease, eye-related infection, and allergy) (16).

Tardive blepharospasm, which is a recognized subtype of TD has rarely reported (17). The pathophysiology of tardive blepharospasm is still unknown. However, some theories suggested that the imbalance of D1 and D2 receptors caused by the long-term use of antipsychotics, subsequently leads to thalamofrontal pathway disinhibition and consequent dystonia (6). A retrospective cohort study found that blepharospasm accounts for $1 \%$ of druginduced TD, with the mixed type being the most common (45\%) followed by orofacial dyskinesia (26\%) (18). The data on the prevalence of tardive blepharospasm associated with a specific antipsychotic treatment is still limited. Several cases of olanzapine-induced blepharospasm have reported (17).

The diagnosis of drug-induced tardive blepharospasm is made clinically and it is very important to rule out the presence of other associated conditions. A thorough history taking with additional physical and neurological examinations can aid the clinician in making a correct diag- 
nosis. Van Egmond et al. (19) proposed a diagnosis algorithm of dyskinesia in children and adolescents, which include stopping the possible offending drug as an early step before proceeding to other specific biochemical tests and magnetic resonance imaging (MRI) of the brain (19). Identifying and diagnosing tardive blepharospasm in patients with schizophrenia is challenging, especially in those with negative symptoms. They may have the lack of spontaneous communication and difficulty in expressing their complaints. In this case, the symptom was first noted by the therapist and only after further clinical history takings, he expressed an unpleasant symptom over both his eyes. Fortunately, the presented sign was identified early, as the delayed recognition of TD can be a risk factor for developing severe and disabling TD (20).

Treatment of TD is mainly symptomatic or empirical, since its pathophysiology is still poorly understood, however various theories have been proposed (1). The American Psychiatric Association Task Force recommends the discontinuation of the offending antipsychotic as soon as TD diagnosis is established, considering the patient's psychosis (21). However, the short-term discontinuation of antipsychotics is associated with the relapse of psychosis and worsening of dyskinesia, particularly in younger patients who are at the higher risk(22) On the other hand, the American Academy of Neurology Guidelines for treatment of TD, published in 2013, states that the evidence of withdrawal of antipsychotics or switching to another antipsychotic with a lower propensity of TD is still insufficient (6). However, clozapine and quetiapine may alleviate the symptoms of TD (23). It has also proposed to consider suppressive therapy by prescribing Clonazepam (2).

Quetiapine has an almost similar pharmacologic profile with clozapine, which may explain the its benefits as a choice of treatment for TD (23). Its relieving effect on TD has thought to be its low affinity for the D2 receptor in the striatal region (24). Besides, another possible mechanism that leads to a lower incidence of TD is its rapid release from dopamine D2 receptors (24). A better phasic dopamine transmission due to the "rapid release effect" subsequently leads to a decline in dyskinesia effect (23). As highlighted in this case, quetiapine was initiated and eventually led to the resolution of the blepharospasm. A similar case of olanzapine-induced tardive blepharospasm was reported and a complete recovery of symptoms was achieved by stopping the offending drug and switching to quetiapine (17).

As the patient stayed for a long time in the hospital, he was seen regularly in the ward throughout his stay and the observable side effect was detected and addressed promptly. However, due to the unavailability of the ophthalmology service in the hospital, assessment by an ophthalmologist was not done during the acute symptoms.
The diagnosis of drug-induced tardive blepharospasm was made clinically by the psychiatrist.

There is limited data available specifically on the reversibility of TD, however the reported remission rate of TD varies between 1 and 62\% (1). As TD is difficult to treat with no definitive treatment, prevention should be highly considered. Therefore, to minimize the risk of developing side effects, the clinician should use the lowest effective dose of antipsychotics and extra caution should be taken regarding the patients at risk for developing TD.

\section{Acknowledgments}

We are thankful to the Director General of Health Malaysia for his permission to publish this article.

\section{Footnotes}

Authors' Contribution: Yih Chew Poh, Arunakiri Muthukrishnan, Yoong Mei Theng collected the data. Yih Chew Poh, Suzaily Wahab, Kalaivani Murugan and Yoong Mei Theng drafted the manuscript. Suzaily Wahab revised critically the manuscript for important intellectual content.

Clinical Trial Registration Code: None declared.

Declaration of Interest: None to declare.

Funding/Support: This study has no funding resource.

Patient Consent: The informed consent was obtained from the patient to publish this report.

\section{References}

1. Cloud LJ, Zutshi D, Factor SA. Tardive dyskinesia: Therapeutic options for an increasingly common disorder. Neurotherapeutics. 2014;11(1):166-76. doi: 10.1007/s13311-013-0222-5. [PubMed: 24310603]. [PubMed Central: PMC3899488].

2. Bhidayasiri R, Jitkritsadakul O, Friedman JH, Fahn S. Updating the recommendations for treatment of tardive syndromes: A systematic review of new evidence and practical treatment algorithm. J Neurol Sci. 2018;389:67-75. doi:10.1016/j.jns.2018.02.010. [PubMed: 29454493].

3. Garcia-Amador M, Merchan-Naranjo J, Tapia C, Moreno C, CastroFornieles J, Baeza I, et al. Neurological adverse effects of antipsychotics in children and adolescents. J Clin Psychopharmacol. 2015;35(6):686-93. doi: 10.1097/JCP.0000000000000419. [PubMed: 26505569].

4. Sobri Muda A, Kwah YG, Al-Edrus SA, Wong SL, Norzaini MZ, Viswanathan S. An unusual cause of blepharospasm. Neuroradiol J. 2010;23(4):443-6. doi: 10.1177/197140091002300413. [PubMed: 24148636].

5. Lee Y, Yeh WC, Chong MY, Lin PY, Chang YY. Venlafaxine and tardive blepharospasm: A case report. Prog Neuropsychopharmacol Biol Psychiatry. 2007;31(5):1139-40. doi: 10.1016/j.pnpbp.2007.03.015. [PubMed: 17478026]. 
6. Bhidayasiri R, Fahn S, Weiner WJ, Gronseth GS, Sullivan KL, Zesiewicz TA, et al. Evidence-based guideline: Treatment of tardive syndromes: Report of the Guideline Development Subcommittee of the American Academy of Neurology. Neurology. 2013;81(5):463-9. doi: 10.1212/WNL.ob013e31829d86b6. [PubMed: 23897874].

7. Taylor DM, Barnes TR, Young AH. The Maudsley prescribing guidelines in psychiatry. New Jersey: John Wiley \& Sons; 2018.

8. Ei Thu H, Hussain Z, Shuid AN. New insight in improving therapeutic efficacy of antipsychotic agents: An overview of improved in vitro and in vivo performance, efficacy upgradation and future prospects. Curr Drug Targets. 2018;19(8):865-76. doi: 10.2174/1389450117666161125174625. [PubMed: 27894237].

9. Said MA, Sulaiman AH, Habil MH, Das S, Bakar AK, Yusoff RM, et al. Metabolic syndrome and cardiovascular risk among patients with schizophrenia receiving antipsychotics in Malaysia. Singapore Med J. 2012;53(12):801-7. [PubMed: 23268153].

10. Adam RL, Sidi H, Midin M, Zakaria H, Das S, Mat KC. The role of atypical antipsychotics in sexuality: Road to recovery in schizophrenia. Curr Drug Targets. 2018;19(12):1402-11. doi: 10.2174/1389450118666170502130126. [PubMed: 28464773].

11. Waln O, Jankovic J. An update on tardive dyskinesia: From phenomenology to treatment. Tremor Other Hyperkinet Mov (N Y). 2013;3. doi: 10.7916/D88P5Z71. [PubMed: 23858394]. [PubMed Central: PMC3709416].

12. Yong KC, Kah TA, Ghee YT, Siang LC, Bastion ML. Branch retinal vein occlusion associated with quetiapine fumarate. BMC Ophthalmol. 2011;11:24. doi: 10.1186/1471-2415-11-24. [PubMed: 21867521]. [PubMed Central: PMC3171721].

13. Glazer WM. Extrapyramidal side effects, tardive dyskinesia, and the concept of atypicality. J Clin Psychiatry. 2000;61 Suppl 3:16-21. [PubMed: 10724129].

14. Kapur S, Zipursky RB, Remington G, Jones C, DaSilva J, Wilson AA, et al. 5-HT2 and D2 receptor occupancy of olanzapine in schizophrenia: A PET investigation. Am J Psychiatry. 1998;155(7):921-8. doi: 10.1176/ajp.155.7.921. [PubMed: 9659858].

15. Wolf DV, Wagner KD. Tardive dyskinesia, tardive dystonia, and tardive Tourette's syndrome in children and adolescents. J Child Adolesc Psychopharmacol.1993;3(4):175-98. doi:10.1089/cap.1993.3.175.
16. Shah H, Yen MT. Management of blepharospasm and hemifacial spasm. Surgery of the eyelids, lacrimal system, and orbit. 2nd ed. New York: Oxford University Press, Inc; 2012. p. 137-44.

17. Arora T, Maharshi V, Rehan HS, Nagar P. Blepharospasm: An uncommon adverse effect caused by long-term administration of olanzapine. J Basic Clin Physiol Pharmacol. 2017;28(1):85-7. doi: 10.1515/jbcpp2016-0037. [PubMed: 27658142].

18. Zutshi D, Cloud LJ, Factor SA. Tardive syndromes are rarely reversible after discontinuing dopamine receptor blocking agents: Experience from a university-based movement disorder clinic. Tremor Other Hyperkinet Mov (N Y). 2014;4:266. doi: 10.7916/D8MS3R8C. [PubMed: 25374768]. [PubMed Central: PMC4219112].

19. van Egmond ME, Kuiper A, Eggink H, Sinke RJ, Brouwer OF, Verschuuren-Bemelmans CC, et al. Dystonia in children and adolescents: A systematic review and a new diagnostic algorithm. J Neurol Neurosurg Psychiatry. 2015;86(7):774-81. doi: 10.1136/jnnp-2014-309106. [PubMed: 25395479].

20. Chandra NC, Sheth SA, Mehta RY, Dave KR. Severe tardive dystonia on low dose short duration exposure to atypical antipsychotics: Factors explored. Indian J Psychol Med. 2017;39(1):96-8. doi: 10.4103/02537176.198938. [PubMed: 28250568]. [PubMed Central: PMC5330001].

21. [No authors listed]. Tardive dyskinesia: Summary of a task force report of the American Psychiatric Association. By the task force on late neurological effects of antipsychotic drugs. Am J Psychiatry. 1980;137(10):1163-72. doi:10.1176/ajp.137.10.1163. [PubMed: 6106389].

22. Gilbert PL, Harris MJ, McAdams LA, Jeste DV. Neuroleptic withdrawal in schizophrenic patients. A review of the literature. Arch Gen Psychiatry. 1995;52(3):173-88. doi: 10.1001/archpsyc.1995.03950150005001. [PubMed: 7872841]

23. Bouckaert F, Herman G, Peuskens J. Rapid remission of severe tardive dyskinesia and tardive dystonia with quetiapine. Int J Geriatr Psychiatry. 2005;20(3):287-8. doi:10.1002/gps.1285. [PubMed: 15770692].

24. Seeman P, Tallerico T. Rapid release of antipsychotic drugs from dopamine D2 receptors: an explanation for low receptor occupancy and early clinical relapse upon withdrawal of clozapine or quetiapine. Am J Psychiatry. 1999;156(6):876-84. doi: 10.1176/ajp.156.6.876. [PubMed: 10360126]. 